kidneys, along with those of macaques, had been used to grow OPV.

Given that OPV was prepared from pools of culture supernatants derived from individual pairs of kidneys, the presence of multiple mitochondrial haplotypes in the same sample is not unexpected. This would also explain the 3:2 mix of M. mulatta and M. fascicularis haplotypes in CHAT type $1 \mathrm{Wy} 4 \mathrm{~B}$. The detection of Homo sequences in CHAT 1FL stems from the fact that the virus was grown on the FL human diploid cell line.

To confirm these findings, we studied a mitochondrial 12S-rRNA locus (101 base pairs) just $5^{\prime}$ to the previous segment. This too was highly informative, with ten point substitutions and one insertion/deletion distinguishing macaque and chimpanzee sequences. All of our findings were confirmed. As the Wistar CHAT pool-13 lot was widely used in Léopoldville, we increased the resolution of the analysis. No chimpanzee sequences were found at a resolution of $1.7 \%$. As this frequency is below the reciprocal of the number of animals generally used to make a lot of OPV, we conclude that none of these OPVs were prepared using chimpanzee kidneys.

In addition, none of the samples was positive when amplified with primers specific for chimpanzee nuclear DNA (data not shown). The extensive use of rhesus and cynomolgus monkeys in the preparation of all the Wistar samples described here not only confirms earlier claims ${ }^{6-8}$, as well as those denying the use of chimpanzee kidneys in the preparation of $\mathrm{OPV}^{9}$, but also reflects the procedures used in the late 1950 s by Salk and Sabin.

CHAT pool 13 was used in Léopoldville between August 1958 and April 1960. The earliest bona fide HIV-1 sequence was derived from a 1959 serum sample taken from an adult living in the suburbs of Léopoldville $^{10}$. According to the OPV/AIDS

\section{Vaccine safety}

\section{Analysis of oral polio vaccine CHAT stocks}

Datches of experimental oral vaccines against poliovirus (OPV CHAT) that were administered in Central Africa in the 1950s have been implicated in the origin of the AIDS pandemic because of possible retroviral contamination during the vaccine's manufacture, which allegedly involved chimpanzee kidney cells ${ }^{1}$. Here we use a molecular analysis to show that two CHAT type-1 polio vaccine stocks were prepared from macaque and not chimpanzee cells, and contain neither human nor simian immunodeficiency virus sequences. Our results do not support the hypothesis that these materials were hypothesis ${ }^{1,2}$, the CHAT pool-13 sample should contain chimpanzee DNA, but as only macaque sequences were found, this tight geographical and temporal association would seem to have been a coincidence.

There is a corollary to our conclusion that the Wistar OPV samples were prepared using macaque kidneys. Given that simian immunodeficiency viruses (SIVs) are confined to African primates, the kidneys could not have been positive for SIV. In other words, the absence of SIV nucleic acids in all samples do not represent false negatives. We find no evidence to support the OPV/AIDS hypothesis. Philippe Blancou*, Jean-Pierre Vartanian*, Cindy Christopherson $\dagger$, Nicole Chenciner ${ }^{*}$, Claudio Basilico $\ddagger$, Shirley Kwok†,

Simon Wain-Hobson ${ }^{*}$

*Unité de Rétrovirologie Moléculaire, Institut Pasteur, 28 rue du Dr Roux, 75724 Paris cedex 15, France e-mail:simon@pasteur.fr

$\dagger$ Department of Infectious Diseases, Roche Molecular Systems, 1145 Atlantic Avenue, Alameda, California 94501, USA

\$Department of Microbiology, New York University School of Medicine, 550 First Avenue, New York, New York 10016, USA

1. Curtis, T. in Rolling Stone 54-60 (1992).

2. Hooper, E. The River: A Journey to the Source of HIV and AIDS (Penguin, London, 1999).

3. Plotkin, S. A., Lebrun, A., Courtois, G. \& Koprowski, H. Bull. World Health Org. 24, 785-792 (1961).

4. van der Kuyl, A. C., Kuiken, C. L., Dekker, J. T. \& Goudsmit, J. J. Mol. Evol. 40, 173-180 (1995).

5. Saitou, N. \& Ueda, S. Mol. Biol. Evol. 11, 504-512 (1994).

6. Koprowski, H. J. Am. Med. Assoc. 178, 1151-1155 (1961).

7. Plotkin, S. A. in Proc. Sixth Intl Congr. Microbiol. Standardisation 48-73 (Hoffman, Berlin, 1961).

8. Plotkin, S. A., Katz, M., Brown, R. E. \& Pagano, J. S. Am. J. Dis. Child. 111, 27-30 (1966).

9. Plotkin, S. A. \& Koprowski, H. Science 286, 2450 (1999).

10. Zhu, T. et al. Nature 391, 531-532 (1998).

11. Hull, R. N., Cherry, W. R. \& Tritch, O. J. J. Exp. Med. 165, 903-917 (1962).

Supplementary information is available on Nature's website at www.nature.com or as paper copy from the London editorial office of Nature. All sequences are available at ftp.pasteur.fr/pub/retromol/ wistar01.

responsible for the transmission of the AIDS virus to humans.

The potential for oral polio vaccines to initiate the AIDS pandemic has been investigated previously ${ }^{2,3}$. Many species of African nonhuman primates are naturally infected with simian immunodeficiency viruses (SIV) and the common chimpanzee (Pan troglodytes) harbours SIV $_{\mathrm{CPZ}}$, the closest relative to modern strains of the human immunodefiency virus HIV-1 (ref. 4). The use of chimpanzee cells to prepare CHAT vaccine may therefore have resulted in the inadvertent transmission of SIV $_{\mathrm{CPZ}}$ to humans.

To test this, we analysed two stocks of OPV CHAT. The first, labelled 19 CHAT 10A-11, was received by NIBSC in 1981 from the Karolinska Institute, Stockholm, and represents an original vial sent from the Wistar

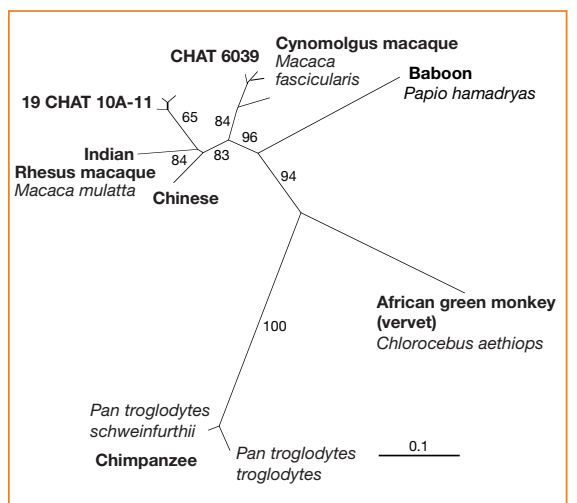

Figure 1 Nucleic-acid sequences were aligned using ClustalW (version 1.5) and evaluated using the Phylip Software package. Evolutionary distances were estimated using DNADIST (default: Kimura 2-parameter method, transition:transversion ratio 2.0) and phylogenetic relationships estimated by the neighbour-joining method using NEIGHBOR. Reproducibility of the branching pattern was assessed with SEQBOOT (boot strap, 100 replicates).

Institute, Philadelphia in 1958. The second, CHAT 6039 (an internal NIBSC number) made at the Institute of Immunology, Zagreb, was received by NIBSC in 1987 . The presence of CHAT virus was confirmed by molecular assay and both samples yielded viable poliovirus in culture as evidence of quality of long-term storage (J. Martin, personal communication).

We tested extracted nucleic acid for HIV-1 RNA using polymerase chain reaction with reverse transcription (RT-PCR) assays based on gag (Amplicor HIV-1 Monitor assay, version 1.5) and combinations of long terminal repeat (LTR) sequences ${ }^{5}$, which are capable of detecting HIV-1 group M (subtypes A-H) ${ }^{6}$, group $\mathrm{N}$ and $\mathrm{O}$ viruses and $\mathrm{SIV}_{\mathrm{CPZ}}$. Additional combinations of LTR sequences used in nested PCR reactions were 507-529 and 524-543 (forward orientation), 622-641 and 628-648 (reverse orientation); the numbering is based on HIV-1 HXB2. HIV-1 primers in pol and env (gp41; ref. 7) and for HIV-2/SIV $\mathrm{SM}_{\text {(ref. 5) }}$ were also included. Sensitivity was shown to be less than 400 RNA equivalents per $\mathrm{ml}$ for each primer set. Using assays that detect this wide range of genetic variants of HIV-1, including SIV $_{\mathrm{CPZ}}$ and the earliest known sequence of HIV-1 present in the Belgian Congo in 1959 (ref. 7), we were unable to find any evidence of HIV/SIV in either CHAT stock.

Substrate cells were identified by targeting the D-loop control region of mitochondrial DNA using PCR. Chimpanzee-specific primers failed to amplify a product from either sample. Furthermore, these primers were capable of detecting limiting amounts of chimpanzee template in the presence of $10^{5}$ macaque-cell equivalents, making it unlikely that chimpanzee cells were used to propagate poliovirus in the two prior passages of vaccine material. However, generic D-loop primers capable of amplifying DNA from baboon, African green monkey 
(vervet), chimpanzee, Chinese and Indian rhesus or cynomolgus macaques successfully yielded product from both batches. Sequencing and phylogenetic analyses indicate that the cells used to prepare 19 CHAT 10A-11 were obtained from rhesus macaques, and those for CHAT 6039 were from cynomolgus macaques (Fig. 1).

Failure to detect HIV/SIV sequences or chimpanzee cellular components in two OPV CHAT stocks, together with the positive identification of macaque mitochondrial sequences, provides no support for the hypothesis that these materials were responsible for the entry of HIV into humans and the source of AIDS.

N. Berry ${ }^{\star}$, C. Davis ${ }^{\star}$, A. Jenkins ${ }^{\star}$, D. Wood $\dagger$, P. Minor $\dagger$, G. Schild $\neq$, M. Bottiger $\$, ~ H$.

Holmes ${ }^{\star}$, N. Almond ${ }^{\star}$

Divisions of ${ }^{\star}$ Retrovirology and $\dagger$ Virology,

$¥$ National Institute for Biological Standards and

Control, Blanche Lane, South Mimms, Potters Bar,

Hertfordshire EN6 3QG, UK

e-mail:nberry@nibsc.ac.uk

\$Karolinska Institute, 17177 Stockholm, Sweden

1. Hooper, E. The River: A Journey to the Source of HIV and AIDS (Penguin, London, 1999)

2. Garrett, A. J., Dunham, A. \& Wood, D. The Lancet 342, 932-933 (1993).

3. Dickson, D. Nature 407, 117 (2000)

4. Hahn, B., Shaw, G., DeCock, K. \& Sharp, P. Science 287, 607-614 (2000).

5. Berry, N. J. et al. J. Hum. Virol. 1, 457-468 (1998).

6. WHO Network for HIV Isolation and Characterisation AIDS Res. Hum. Retrovir. 10, 1327-1343 (1994).

7. Zhu, T. et al. Nature 391, 594-597 (1998)

\section{Human immunodeficiency virus}

\section{Phylogeny and the origin of HIV-1}

- he origin of human immunodeficiency virus type 1 (HIV-1) is controversial. We show here that viruses obtained from the Democratic Republic of Congo in Africa have a quantitatively different phylogenetic tree structure from those sampled in other parts of the world. This indicates that the structure of HIV-1 phylogenies is the result of epidemiological processes acting within human populations alone, and is not due to multiple cross-species transmission initiated by oral polio vaccination.

According to the oral polio vaccination (OPV) hypothesis, the main (M) group of HIV-1 (the viruses responsible for the majority of global AIDS cases) emerged as a result of the vaccination of about one million people, who were largely living in the Congo from 1957-60, with an oral vaccine against polio virus that had allegedly been cultured in chimpanzee kidneys ${ }^{1}$. This is claimed to have enabled the transfer to humans of chimpanzee simian immunodeficiency virus, the closest relative of HIV-1.

Conversely, phylogenetic analysis of HIV-1 sequences indicates that group $\mathrm{M}$ originated

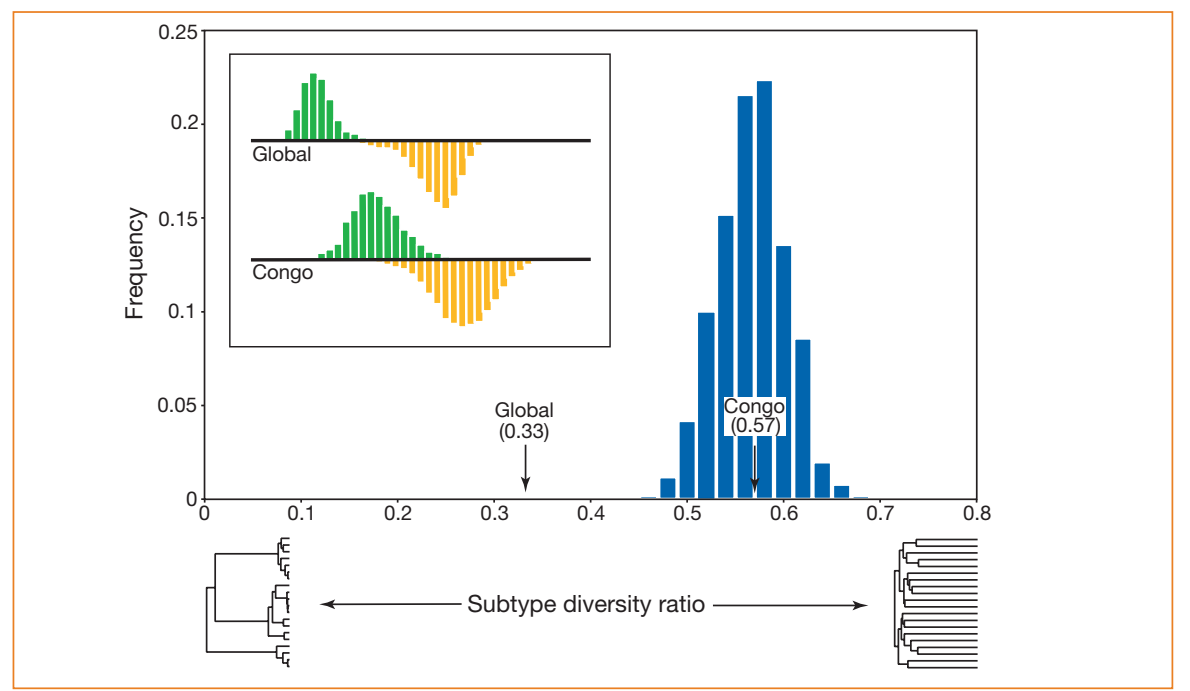

Figure 1 Maximum-likelihood phylogenies were estimated for three V3-V5 data sets: HIV-1 sequences from the Democratic Republic of Congo (423 base pairs), global isolates (426 base pairs), and Congo and global isolates combined (396 base pairs). Given a phylogeny with tips labelled according to subtype, the subtype diversity ratio (SDR) was calculated as the mean path length between tips of the same subtype divided by the mean path length between tips of different subtypes. For the phylogeny of the global isolates, 11 subtypes were allocated according to standard HIV-1 nomenclature ${ }^{7}$. For the Congo phylogeny, 11 subtypes were allocated so as to minimize the SDR score, using a heuristic optimization algorithm. This assignment is that which gives the maximum possible subtype structure for the Congo phylogeny. The global phylogeny gave an SDR of 0.33 and the Congo a value of 0.57 . The analysis was repeated after removal of the Congo and global sequences previously identified as intersubtype recombinants ${ }^{4,5}$. Our analysis will only be affected if recombination breakpoints fall within the V3-V5 region, so excluding recombinants changes the SDR only marginally ( 0.35 for the global phylogeny; 0.58 for the Congo). SDR values were similar when Congo isolates were assigned to different numbers of subtypes (for example, 0.59 and 0.55 in the case of 8 and 14 subtypes, respectively). To assess the significance of the difference between the global and Congo SDRs, we obtained a null distribution by simulating phylogenies under an exponential growth coalescent process inferred from env gene sequences of subtype A (ref. 6), which is common in Africa. The frequency distribution of minimum SDR values for these simulated phylogenies is shown in blue. Inset: normalized frequency distributions of intrasubtype path lengths (above the line) and intersubtype path lengths (below the line), plotted on the same horizontal scale (0.0-0.8 substitutions per site), for the global and Congo phylogenies. See supplementary information for details of trees and phylogenetic methods.

before the vaccination campaign ${ }^{2}$, supporting a model of 'natural transfer' from chimpanzees to humans ${ }^{3}$. If this timescale is correct, then the OPV theory remains a viable hypothesis of HIV-1 origins only if the subtypes of group $\mathrm{M}$ differentiated in chimpanzees before their transmission to humans.

It has been suggested that the distinctive structure of the global group-M tree, which has been called a 'starburst' because of the apparently simultaneous appearance of viral subtypes, is consistent with the transfer of multiple viral lineages from chimpanzees to humans ${ }^{1}$. To test this, we analysed partial env sequences (V3-V5) of 197 HIV-1 isolates sampled in 1997 from the Congo ${ }^{4}$, a likely location for the origin of HIV-1 group M under both hypotheses.

A phylogeny comprising the Congo data, plus 223 sequences representing the global diversity of HIV-1 (including all known subtypes), reveals comparable genetic diversity in the Congo strains to that among global strains, with many Congo lineages falling basal to the origin of each subtype as currently defined by the phylogeny of global strains ${ }^{5}$. We tested whether the structure of the Congo phylogeny differed from that of the global HIV-1 M group by comparing the subtype diversity ratio (SDR) of the two phylogenies (Fig.1). This is defined as the ratio of the mean within-subtype pairwise distance to the mean between-subtype pairwise distance.

Rather than assigning the Congo isolates to subtypes by their phylogenetic relationship to global strains, we used a heuristic algorithm to assign subtypes such that the subtype diversity ratio was minimized. The Congo and global phylogenies differ significantly in the SDR statistic, with the former showing no more subtype structure than phylogenetic trees simulated under a model of exponential population growth ${ }^{6}$ (Fig. 1; see supplementary information). This result is conservative because the minimum possible ratio value (representing maximum subtype structure) was used in the Congo analysis. Furthermore, although subtypes can be clearly identified in the distribution of pairwise distances for the global sequences (Fig. 1, inset), there is much less distinction between intra- and intersubtype comparisons for the Congo sequences. Hence, for any two randomly chosen Congo sequences, it is difficult to determine unambiguously whether they belong to the same or to different subtypes.

Our results indicate that the Congo and global phylogenies probably result from different epidemiological histories. As many Congo strains appear to be basal, we propose that each global subtype is the result of the chance exportation of some Congo strains to other geographical regions, thus producing an apparent starburst. Such founder effects have 